\title{
Resonant Type Piezoelectric Pump Driven at the Power Frequency Using a Tuning Fork Vibrator
}

\author{
Qiaosheng Pan, Haiyang Jiang, Yinghao Li, Quan Wang, Bin Huang and Ruijun Li \\ School of Instrument Science and Opto-Electronics Engineering, Hefei University of Technology, Hefei, Anhui \\ 230009, China.
}

\author{
Zhihua Feng \\ Department of Precision Machinery and Precision Instrumentation, University of Science and Technology of \\ China, Hefei, Anhui 230026, China.
}

\begin{abstract}
(Received 19 April 2020; accepted 5 October 2020)
A resonant type piezoelectric pump driven by a tuning fork vibrator (TFV) is developed in this study. The resonant frequency of the TFV is designed to be the power frequency, thus the pump can directly work in a resonant state under a power frequency power supply. Three factors affecting the performance of the piezoelectric pump are investigated in this paper, including the thickness of the TFV, the material of the pump chamber diaphragm and the quantity of inlet valve. The Taguchi method is adopted to obtain the best combination among the three factors. In accordance with the optimized results of the Taguchi method, a final prototype piezoelectric pump is fabricated via $3 \mathrm{D}$ printing technology. When driven by the power frequency power supply, the maximum flow rate of the prototype piezoelectric pump can reach $441.77 \mathrm{ml} / \mathrm{min}$.
\end{abstract}

\section{INTRODUCTION}

As a new type of fluid driver that exhibits superior performance, piezoelectric pumps have received considerable attention because of their small size, low power consumption and anti-electromagnetic interference. ${ }^{1-3}$ The demand for the application of simple micropumps with a high flow rate and strong pumping pressure that are lightweight and economical has increased in recent years in the fields of chemical analysis, medical devices, fuel cells and microelectronic devices. ${ }^{3,4}$

From the perspective of energy conversion, a piezoelectric pump is composed of an excitation source, a vibrator and a chamber. The excitation source provides electrical energy and the piezoelectric element attached to the vibrator converts the electrical energy into mechanical energy. The vibrator produces the deformation and transmits it to the pump chamber diaphragm, causing a volume change in the pump chamber. Finally, the pump chamber performs work on the water to complete the pumping process. In this manner, the vibrator plays a crucial role in the entire system. The optimization and improvement of the vibrator can significantly improve the performance of the pump. Scholars have conducted considerable research on vibrators for decades. The original vibrator structure consisted of a piezoelectric sheet attached to the pump chamber diaphragm. ${ }^{5}$ This type of vibrator has a low operating frequency and experiences difficulty in exerting the advantage of high power density of piezoelectric materials. ${ }^{6,7}$ A longbeam vibrator operating in a resonant state was proposed by Wang et al. ${ }^{8}$ Under an excitation of $490 \mathrm{~Hz}$ and an AC voltage of $400 \mathrm{~V}$, the flow rate of the piezoelectric pump reached $105 \mathrm{ml} / \mathrm{min}$. Subsequently, a multilayer folded vibrator was produced. Under an excitation of $361 \mathrm{~Hz}$ and an AC voltage of
$120 \mathrm{~V}$, the flow rate of the piezoelectric pump was $118 \mathrm{ml} / \mathrm{min}$. A U-shaped vibrator that simultaneously drives two pumps was proposed by Chen et al. ${ }^{9}$ in 2015 . However, this pump requires a specialized power supply, which makes the entire structure complicated, increasing its cost and causing inconvenience in the operation process. Pumps that work under a common power supply emerged in the market to simplify the drive power supply and make the whole structure simpler.

This study develops a new resonant-type piezoelectric pump driven at the power frequency. It also designs a vibrator within the tuning fork structure. The resonant frequency of the TFV is set to $50 \mathrm{~Hz}$ (Power frequency in China) by adjusting its structure and size. Therefore, the resonant state of the TFV can be excited when driven by a power frequency power supply. The Taguchi method has been adopted to optimise and improve the performance of the piezoelectric pump. In accordance with the optimised results of the Taguchi method, a final prototype piezoelectric pump is fabricated and tested. Experimental results show that the performance of the pump has been improved. Section 2 describes the working principle of the piezoelectric pump. Section 3 presents the design and fabrication processes of the TFV and the entire pump. Section 4 presents the detailed process of the Taguchi method and the performance of the final prototype piezoelectric pump.

\section{WORKING PRINCIPLE}

The working principle of the proposed piezoelectric pump is illustrated in Figs. 1(a) and 1(b). The piezoelectric pump consists of four parts: a piezoelectric actuator (tuning fork vibrator and piezoelectric sheet), a pump chamber, check valves (inlet valve and outlet valve), and flow channels. The piezoelec- 


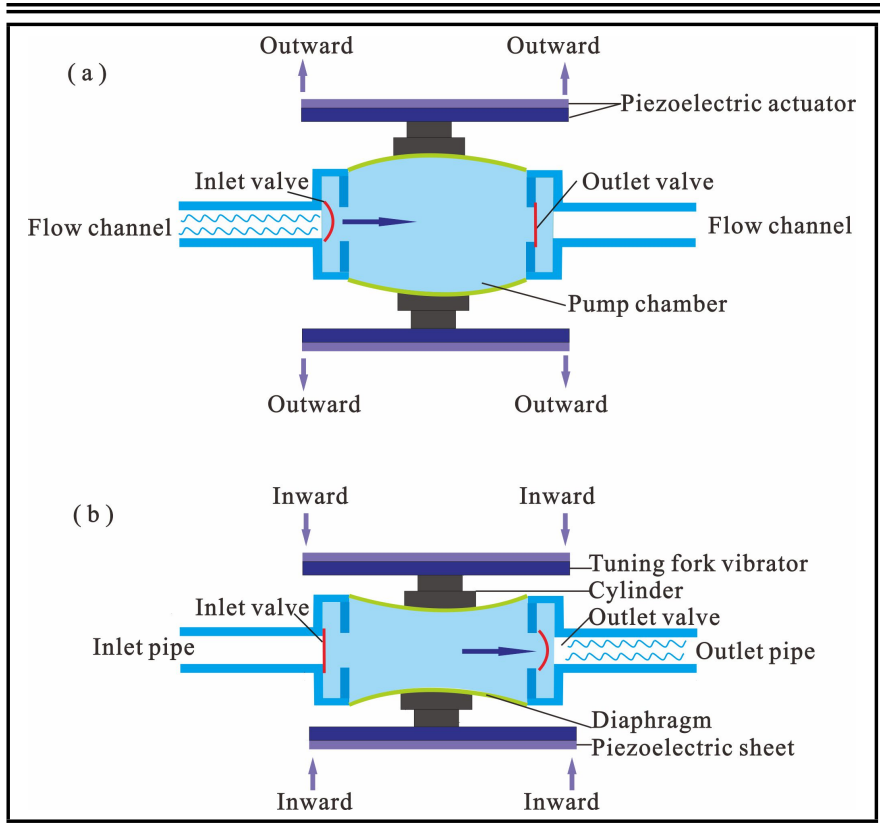

Figure 1. Working principle of the proposed piezoelectric pump: (a) Schematic diagram of absorbing mode (b) Schematic diagram of dispensing mode.

tric actuator generates vibration controlled by the excitation of the power source. Vibrations are passed from the piezoelectric actuator to the diaphragm through a cylinder. As shown in Fig. 1(a), when the piezoelectric actuator moves outward, the volume of the chamber increases and internal pressure decreases. The inlet valve is opened and the outlet valve is closed, the fluid is driven into the pump chamber by the differential pressure. This process is referred as water intake (absorbing mode). As shown in Fig. 1(b), when the piezoelectric actuator moves inward, the volume of the chamber decreases and internal pressure increases. The inlet valve is closed and the outlet valve is opened. The fluid is driven outside the pump chamber by differential pressure. This process is referred as water outflow (dispensing mode). A steady stream of water can be achieved with this cycle.

In accordance with theory of vibration, a single-prong tuning fork vibrator (TFV) can be regarded as a Euler-Bernoulli beam with one end fixed and one end free. The vibration amplitude generated at the resonant frequency of the anti-phase mode is the largest. ${ }^{10}$ On the basis of the force balance and moment equations, the resonant frequency of the anti-phase mode of the TFV is: ${ }^{11}$

$$
f_{1}=\frac{1.875^{2}}{2 \pi L_{0}^{2}} \sqrt{\frac{E_{1} I_{1}}{\rho_{1} S}}
$$

where $E_{1}$ is the elastic modulus of the tuning fork material, $I_{1}$ is the moment of inertia of the section to the centre, $\rho_{1}$ is the density of the tuning fork material, $S$ is the cross-sectional area of the Euler-Bernoulli beam, $L_{0}$ is the length of the tuning fork prong. An arbitrary resonant frequency TFV can be designed by adjusting these parameters $\left(E_{1}, I_{1}, \rho_{1}, S, L_{0}\right)$. Equation (1) has a qualitative guiding significance for modifying geometric dimensions of TFV to achieve the target frequency design of $50 \mathrm{~Hz}$.

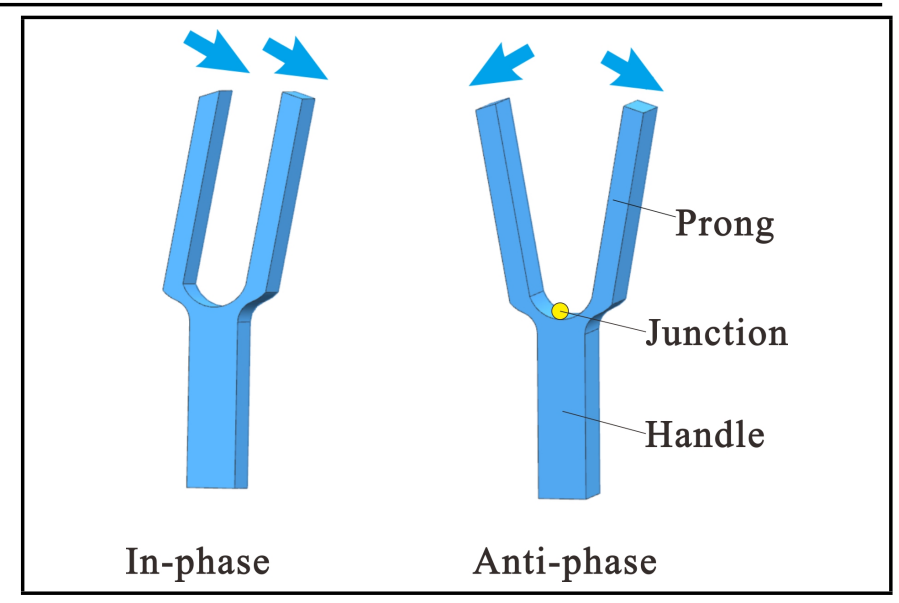

Figure 2. Two vibration modes of the tuning fork vibrator.

Table 1. Properties of the materials.

\begin{tabular}{||l|l|l|l|}
\hline Material & $\begin{array}{l}\text { Density } \\
\left(\mathrm{Kg} \mathrm{m}^{-3}\right)\end{array}$ & $\begin{array}{l}\text { Young's } \\
\text { Modulus }(\mathrm{GPa})\end{array}$ & $\begin{array}{l}\text { Poisson's } \\
\text { ratio }\end{array}$ \\
\hline PZT- 4 & 7500 & 79 & 0.31 \\
\hline 65 Manganese steel & $7800\left(\rho_{1}\right)$ & $200\left(E_{1}\right)$ & 0.30 \\
\hline
\end{tabular}

\section{DESIGN AND FABRICATION}

\subsection{Design and Fabrication of the TFV}

This study designed a vibrator within the structure of a tuning fork. The TFV has two vibration modes, namely, in-phase and anti-phase modes, as shown in Fig. 2. In its anti-phase mode, ${ }^{12,13}$ the bending stress of the tuning fork prong cancels out at the junction (Fig. 2) of the two tuning fork prongs. The energy of the vibration can be maintained between the two tuning fork prongs and is not easily transmitted to the tuning fork handle and the outside area. Therefore, the anti-phase mode can maintain a high energy conversion efficiency. ${ }^{14-16}$ The anti-phase mode of the TFV was utilized in our design.

To develop a TFV with a compact structure, a mass block (Fig. 3(a)) at the end of the tuning fork prong is designed. This new structure can increase the output force of the TFV, which is beneficial for the force matching between the TFV and the pump chamber.

The finite element analysis software ANSYS was used to realize the frequency design of the TFV. The process of modal analysis includes 3D modelling, definition of material parameters, meshing and calculation of resonant frequency. Under the guidance of Eq. (1), three types of TFV with typical dimensions were designed, as shown in Figs. 3(a), 3(b) and 3(c). In simulation, the length $(L)$ and width $(W)$ of the mass block were constantly adjusted to achieve the design of the target frequency $(50 \mathrm{~Hz})$. The TFV was made of 65 manganese steel and the piezoelectric sheet was made of PZT-4S (Hunan Jiayeda Electronics Co., Ltd.). ${ }^{17}$ The material parameters of 65 manganese steel and PZT-4S are listed in Table 1. The density and Young's modulus of 65 manganese steel in Table 1 correspond to $E_{1}$ and $\rho_{1}$ in Eq. (1), respectively.

The frequency-length and frequency-width curve shown in Figs. 3(d) and 3(e) were obtained via simulation. The results show that an inverse correlation exists between the resonant frequency and the length $(L)$ and the width $(W)$. Three TFVs 


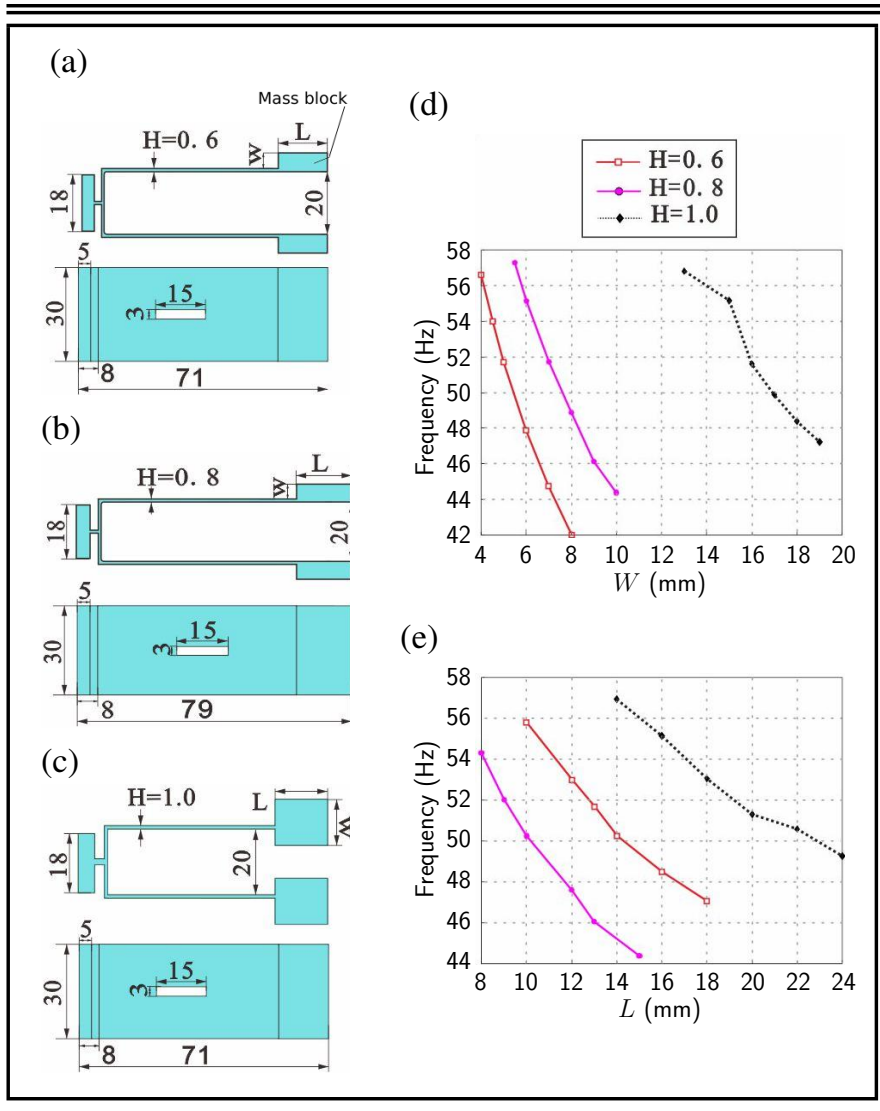

Figure 3. Simulation results: (a) dimensions of TFV 1; (b) dimensions of TFV 2; (c) dimensions of TFV 3; (d) relationship between the thickness of the mass block $(W)$ and the resonant frequency; (e) relationship between the width of the mass block $(L)$ and the resonant frequency.

that satisfy the conditions were obtained via simulation. TFV 1 ( $L=13 \mathrm{~mm}, W=5 \mathrm{~mm})$, TFV $2(L=15 \mathrm{~mm}, W=10 \mathrm{~mm})$ and TFV $3(L=16 \mathrm{~mm}, W=14 \mathrm{~mm})$ were designed and manufactured using a wire-cutting process (with an accuracy of $0.1 \mathrm{~mm})$.

As illustrated in Fig. 4, the resonant frequency of the TFV was adjusted to $50.2 \mathrm{~Hz}$ which is the key parameter in ANSYS simulation. The TFV exhibits good vibration symmetry, thus ensuring that the two prongs reach maximum deformation simultaneously to achieve excellent performance of the tuning fork.

The well-fixed TFV was connected to the LCR metre. The test voltage was set at $1 \mathrm{~V}$ and the range of excitation frequency was set from $30 \mathrm{~Hz}$ to $80 \mathrm{~Hz}$. Deviations were detected between the simulation and measured results of the resonant frequency in the process of testing due to the deviation between the actual and theoretical parameters of the selected materials and the objective existence of machining error. Deviations will affect pump performance. The magnets were adjusted to different sizes and attached at different positions of the TFV to fine tune the deviation. Finally, the resonant frequency of the tuning fork vibration was adjusted to $50 \mathrm{~Hz}$ as shown in Fig. 5.

\subsection{Pump Fabrication}

Figure. 6(a) shows the exploded view of the entire piezoelectric pump. The entire pump is primarily made of high temperature-resistant nylon, which is processed via 3D printing technology. The manufacturing process of the entire device

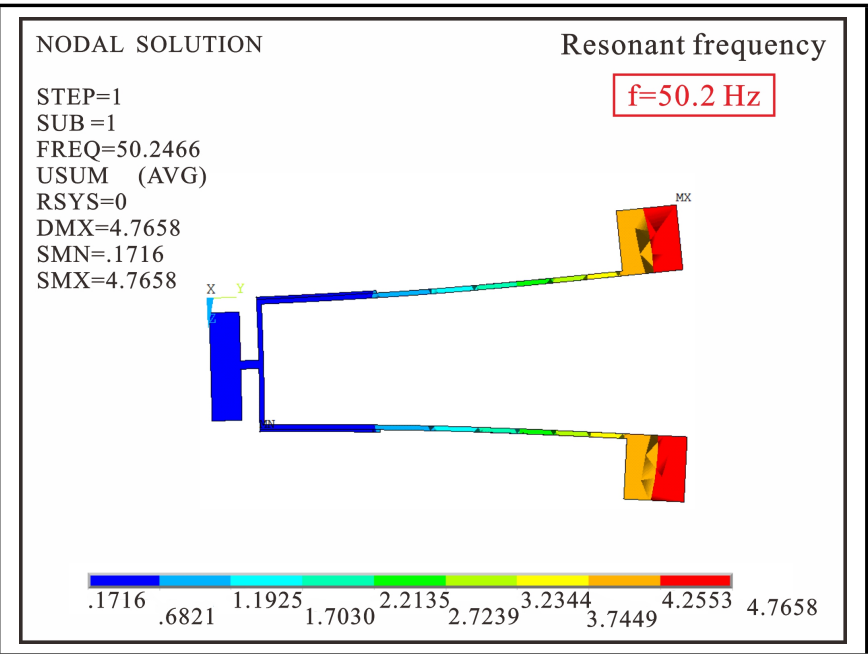

Figure 4. Simulation results of TFV resonant frequency.

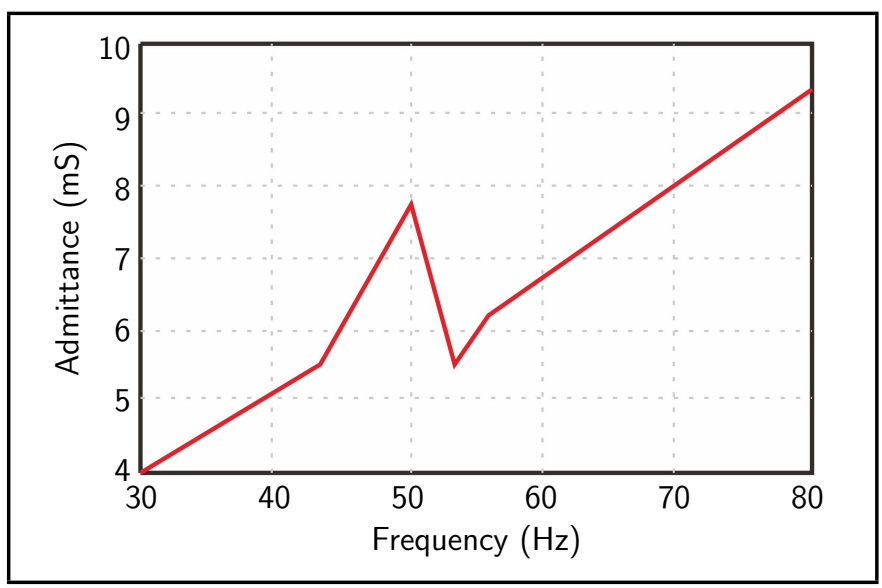

Figure 5. Test result of LCR meter.

is discussed in detail in the following steps.

(1) The pump chamber was fabricated. The inlet and outlet seat plate (Fig. 6(a), part 7, with a dimension of $40 \mathrm{~mm}$ $\times 20 \mathrm{~mm} \times 6 \mathrm{~mm}$ ) sandwiched between two diaphragms (Fig. 6(a), part 5) comprises the pump chamber. Throughout the experiment, the pump chamber diaphragms were sequentially made of three materials: rubber, PDMS and polyimide, which achieved a different experimental purpose.

(2) The inlet and outlet valves were fabricated. As shown in Fig. 7, the check valve with a shape similar to a 'double H' was cut from a $0.3 \mathrm{~mm}$-thick polydimethylsiloxane (PDMS) film. The valve is slightly larger than the valve hole. The narrow sides of the entire valve were designed to make rushing through easier for water. The check valve was attached on the inlet and outlet valve seat plate using cyanoacrylate adhesive.

(3) The pump was fabricated as follows:

1.) two pump chamber diaphragms were glued to the inlet and outlet valve seat plate using cyanoacrylate adhesive,

2.) the cover plate pressed the diaphragm firmly onto the inlet and outlet valve seat plate, 


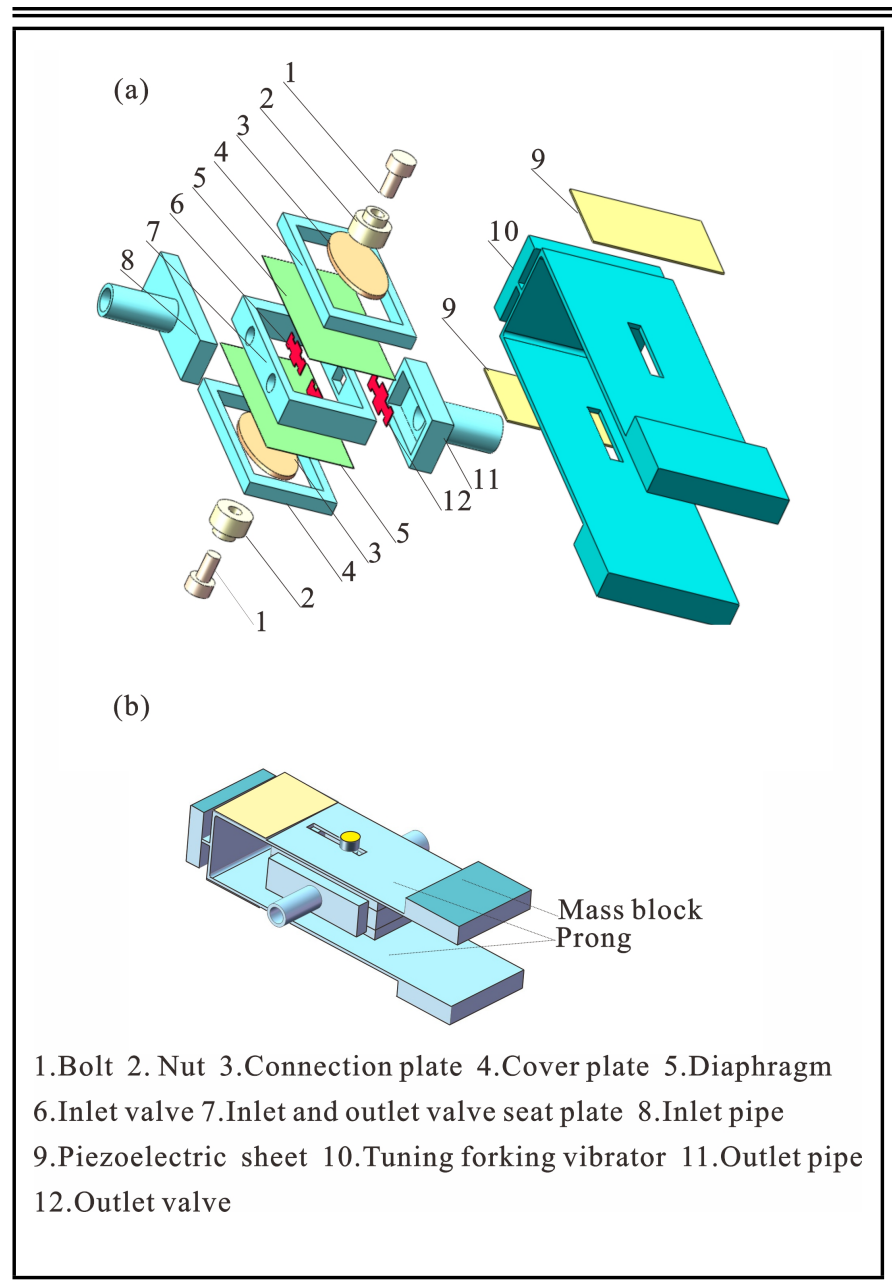

Figure 6. (a) Exploded view of the designed piezoelectric pump (b) Rendering of the piezoelectric pump.

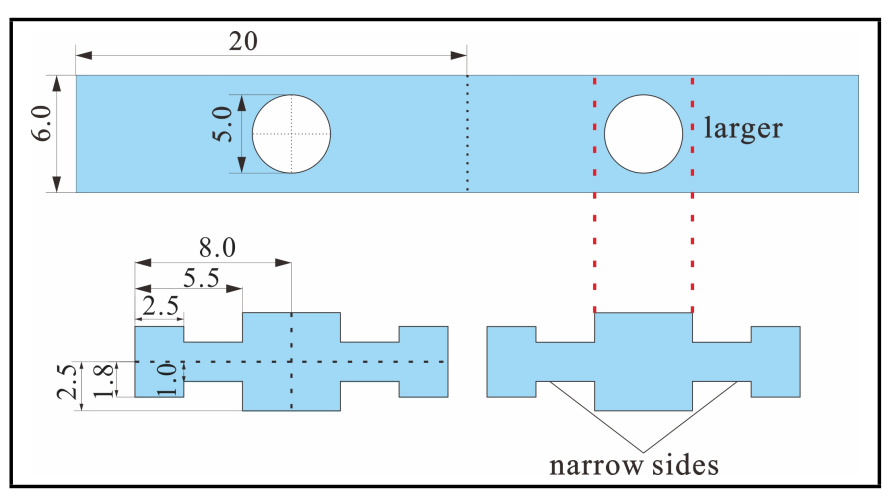

Figure 7. Relative position of check valve and valve hole.

3.) the centre of the inlet and outlet pipes was aligned with the valve hole,

4.) glue (DP460) was applied evenly to the contact area of the aforementioned primary parts (Fig. 6(a); parts $4,7,8$ and 11) and,

5.) the glued pump was placed in a $60{ }^{\circ} \mathrm{C}$ incubator for $2 \mathrm{~h}$ to achieve a good sealing effect.

(4) The connection plate was glued to the centre of the diaphragm using cyanoacrylate adhesive. A slit was made on the TFV to transfer the deformation generated by the TFV to the pump chamber diaphragm. Subsequently, the two parts (TFV and pump) were fixed together with bolts

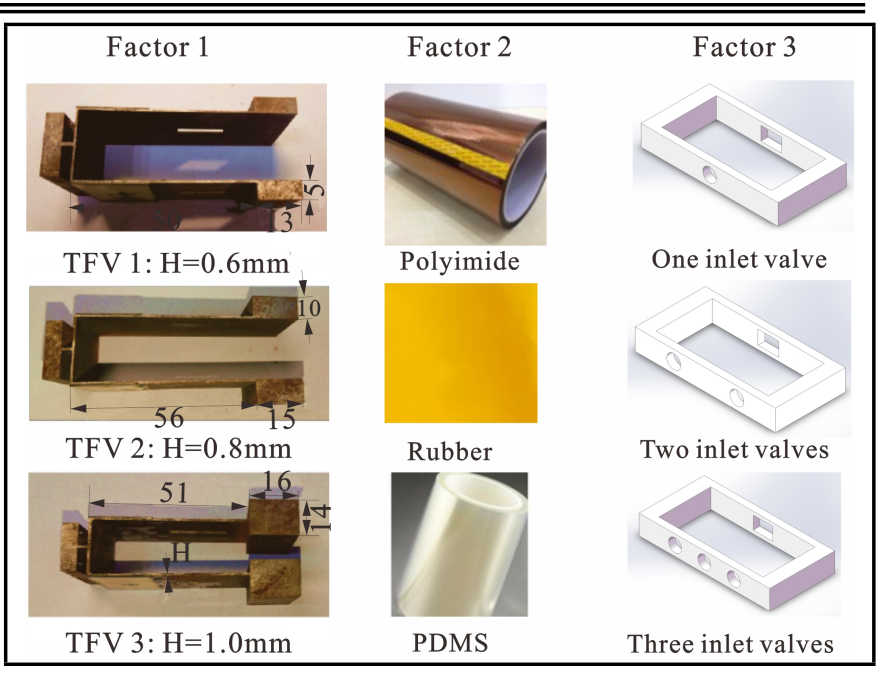

Figure 8. Three primary factors affecting the performance of the piezoelectric pump.

and nuts. Figure 6(b) presents the rendering of the entire device. It should be noted that the working principle of the proposed piezoelectric pump shown in Fig. 1 is viewed from the right view direction of Fig. 6(b).

\section{EXPERIMENTAL DISCUSSION \\ 4.1. Experimental Design: The Taguchi Method}

This study examined three factors affecting the performance of the piezoelectric pump, including the thickness of the TFV (Factor 1), the material composition of the pump chamber diaphragm (Factor 2) and the quantity of the inlet valve (Factor 3) as illustrated in Fig. 8. Piezoelectric pumps are driven by energy. These three factors play important roles in the generation, transmission and action of energy. For Factor 1, theoretically, the thickness of the TFV can be an arbitrary value to achieve the target frequency design of $50 \mathrm{~Hz}$. However, a TFV whose thickness is greater than $1.0 \mathrm{~mm}$ while maintaining the resonance frequency at $50 \mathrm{~Hz}$ will make the whole volume of the TFV larger. In addition, the matching effect of the TFV and pump chamber may become difficult when the thickness of TFV is greater than $1.0 \mathrm{~mm}$. Three typical thickness values that can realize the target frequency design of $50 \mathrm{~Hz}$ while maintaining a small volume of the TFV are 1.0, 0.8, and $0.6 \mathrm{~mm}$. Therefore, the thickness of TFV is determined to be $0.6,0.8$ and $1.0 \mathrm{~mm}$ in current study.

The Taguchi method ${ }^{18,19}$ was used for experimental research and optimization to obtain the best combination among the aforementioned three factors. The steps of the Taguchi method include designing a three-factor orthogonal array (Table 2), conducting nine groups of experiments and drawing a primary effect diagram according to the experimental results. Flow rate was selected as the characteristic parameter for judging the performance of the piezoelectric pump. Designing Taguchi's orthogonal array and analyzing the primary effect diagram can obtain the best combination among multiple factors with the least number of experiments. The primary effect diagram is 
Table 2. Three-factor orthogonal array of the Taguchi method.

\begin{tabular}{||l|l|l|l||}
\hline $\begin{array}{l}\text { Number of } \\
\text { experiments }\end{array}$ & $\begin{array}{l}H \\
(\mathrm{~mm})\end{array}$ & Material & $\begin{array}{l}\text { Quantity of } \\
\text { inlet valve }\end{array}$ \\
\hline 1 & 1.0 & Polyimide & 1 \\
\hline 2 & 1.0 & Rubber & 2 \\
\hline 3 & 1.0 & PDMS & 3 \\
\hline 4 & 0.6 & Polyimide & 2 \\
\hline 5 & 0.6 & Rubber & 3 \\
\hline 6 & 0.6 & PDMS & 1 \\
\hline 7 & 0.8 & Polyimide & 3 \\
\hline 8 & 0.8 & Rubber & 1 \\
\hline 9 & 0.8 & PDMS & 2 \\
\hline
\end{tabular}

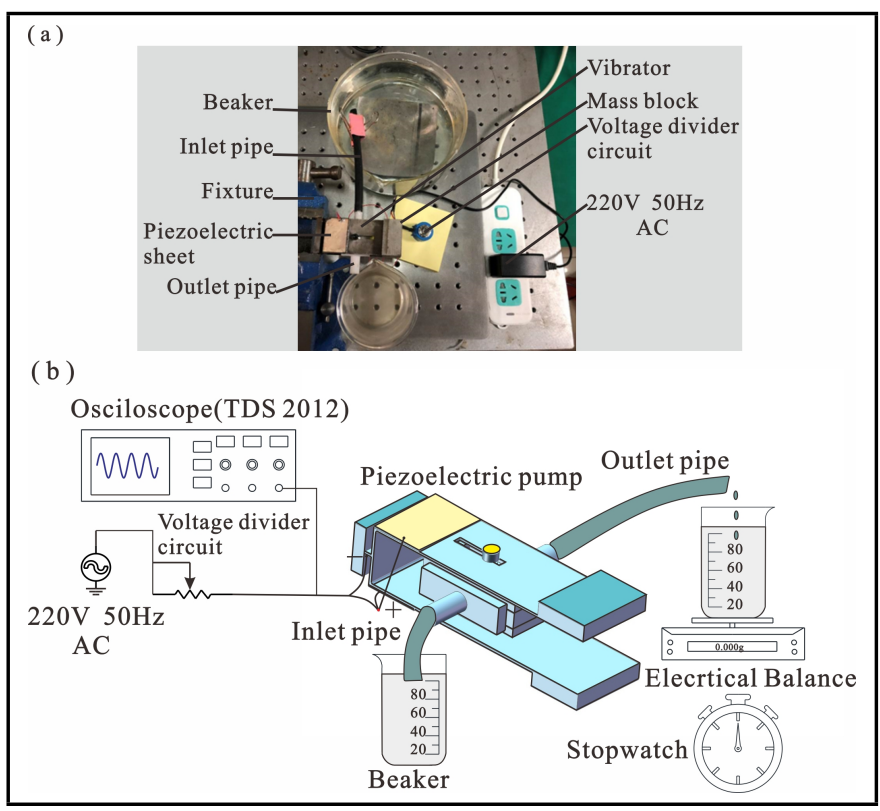

Figure 9. Experimental setup for the piezoelectric pump: (a) Physical figure (b) Rendergraph.

used to determine which amongst the three factors exerts the most influence. The factor whose flow rate and signal-to-noise ratio are highest exerts the most influence on the performance of the pump.

\subsection{Experimental Setup and Datas}

An experimental setup (shown in Fig. 9) was built to investigate the performance of the piezoelectric pump. The entire experimental system is primarily composed of five parts: 1.) power frequency power supply, 2.) piezoelectric pump, 3.) oscilloscope, 4.) water holding device, and 5.) flow rate measurement device. A partial voltage divider circuit was connected to adjust the amplitude of the output voltage. To ensure the accuracy of the experimental results, three to five measurements were taken for each group of experiments during the test and the average value was taken as the final result shown in Fig. 10.

The signal-to-noise ratio $(\mathrm{S} / \mathrm{N})$, as shown in Fig. 11(b), is an evaluation criterion for the robustness of a system. Given that $\mathrm{S} / \mathrm{N}$ is expected to be larger, a large $\mathrm{S} / \mathrm{N}^{20,21}$ in Eq. (2) is adopted.

$$
\eta=-10 \log \frac{1}{n}\left(\sum \frac{1}{y_{i}^{2}}\right)
$$

where $n$ is the number of experiments, and $y_{i}$ is the flow rate of the experiment in Group $i$.

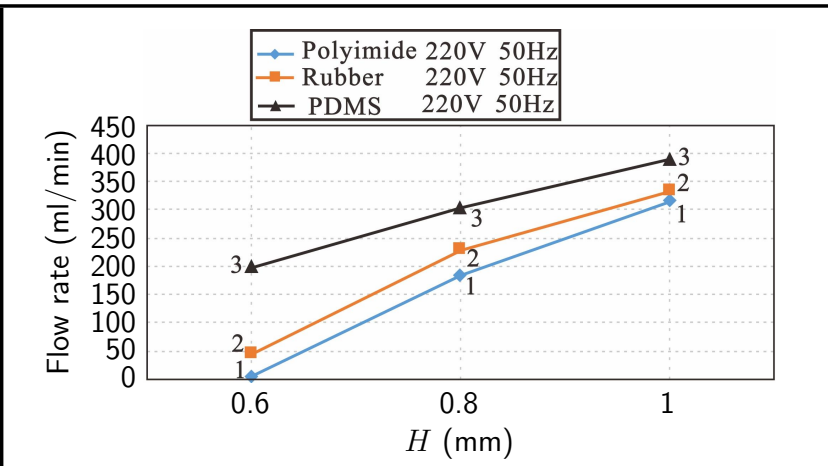

1: One inlet valve; 2: Two inlet valves; 3 : Three inlet valves

Figure 10. Results of the nine groups of experiments.

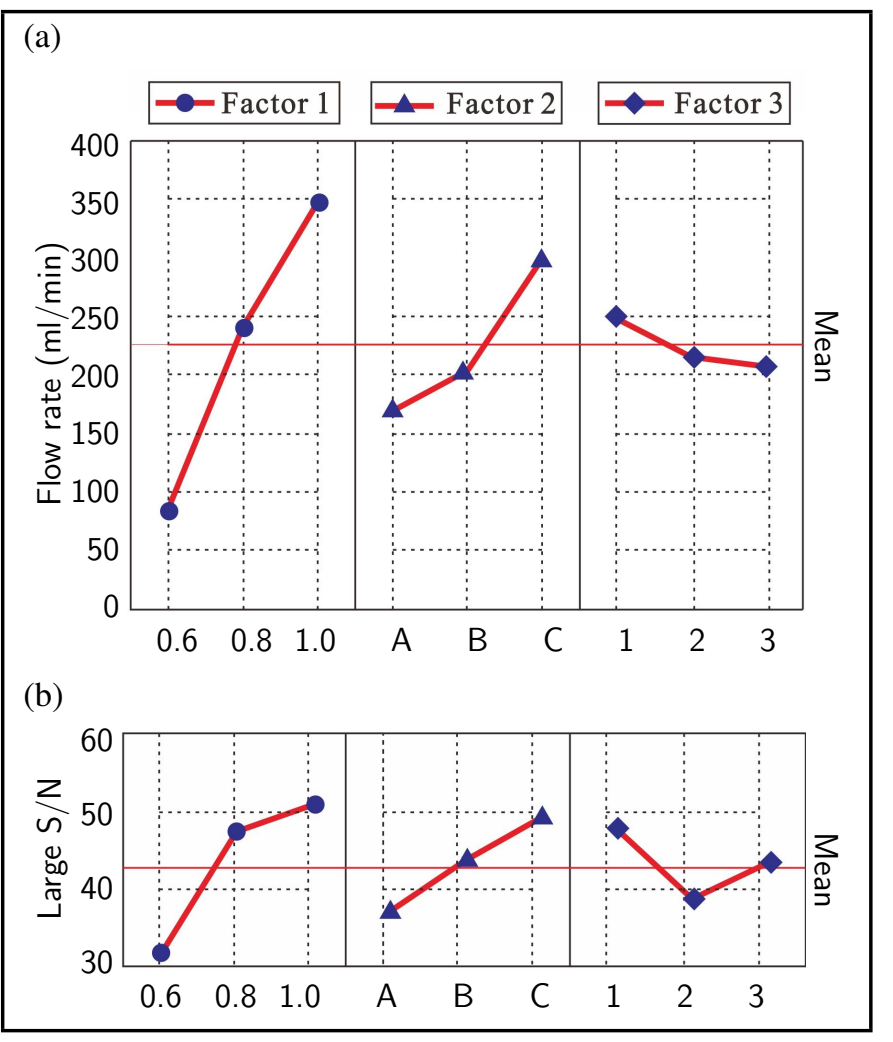

Figure 11. (a) Primary effect diagram of the flow (b) Primary effect diagram of large S/N. Factor 1: thickness of vibrator: $0.60 .81 .0(\mathrm{~mm})$; Factor 2: material of pump chamber diaphragm, A: Polyimide B: Rubber C: PDMS; Factor 3: quantity of inlet valve: 123 .

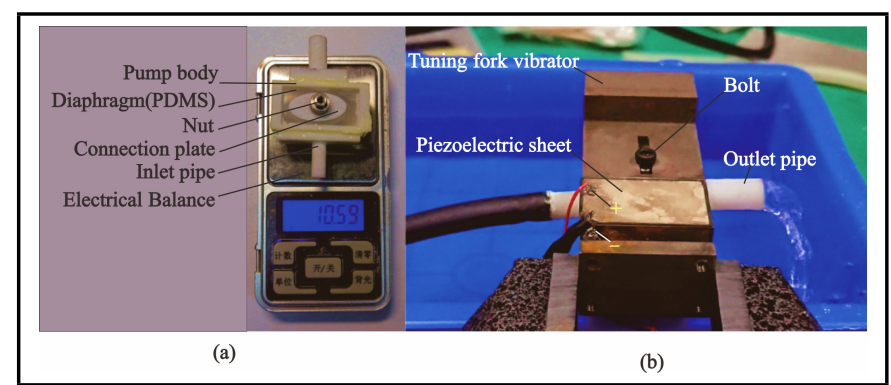

Figure 12. (a) Pumps made via 3D printing technology (b) Piezoelectric pump under testing. 
The experimental results in Figs. 11(a) and 11(b) show that the magnitude of Factor 1 has the highest flow rate and signalto-noise ratio, followed by Factors 2 and 3. Therefore, Factor 1 has the most significant effect on flow rate, followed by Factors 2 and 3 .

\subsection{Discussion}

\subsubsection{Factor 1}

Factor 1 and the flow rate present a positive correlation. It can be observed from Fig. 11 that ' $1.0 \mathrm{~mm}$ ' has the highest flow rate and its signal-to-noise ratio is the highest in Factor 1. When the thickness of the TFV is $1.0 \mathrm{~mm}$, the matching effect of the output force and amplitude of the TFV is the best in Factor 1.

\subsubsection{Factor 2}

The stiffness of Factor 2 exhibits an inverse correlation with flow rate. Piezoelectric pumps rely on the vibration of the elastic pump chamber diaphragm during the working process, causing periodic changes in the volume of the pump chamber. The stiffness of "PDMS" is minimal in Factor 2. Under the same force, it can cause the largest change in the volume of the pump chamber, which can produce the largest flow rate.

\subsubsection{Factor 3}

It can be observed from the primary diagram shown in Fig. 11 that 'One inlet valve' has the highest flow rate and its signal-to-noise ratio is the hignest in Factor 3. This conclusion may subvert the conventional perception that multiple inlet valves produce higher flow rate. When the fluid is driven into the pump chamber through multiple inlet valves at the same time, the disturbance between multiple inlet valves may cause flow energy loss. The liquid flow direction may be linear and the flow energy loss is minimal when the quantity of inlet valve is one. Thus, the piezoelectric pump with one inlet valve obtains the largest flowrate.

\subsection{Flow Rate Characteristics of Final Prototype Piezoelectric Pump}

On the basis of the analysis results in the primary effect diagram, a final prototype piezoelectric pump was built as shown in Fig. 12, and its weight was $10.59 \mathrm{~g}$. The frequency characteristics of the pump were measured using a signal generator to provide a variable frequency for the pump's drive source. The driving voltage was set to $220 \mathrm{~V}$ (effective value) in the experiment. To show performance parameters of the piezoelectric pump in detail, the pump performance (flow rate) under the driven frequencies of $42-58 \mathrm{~Hz}$ is also given in this paper. The maximum flow rate reached $441.77 \mathrm{ml} / \mathrm{min}$ when the resonant frequency was $50 \mathrm{~Hz}$, which also verifies that the resonant state is achieved under the power frequency drive.

The frequency of the driving voltage was set to $50 \mathrm{~Hz}$ and the amplitude of the driving voltage was gradually increased to obtain the curve shown in Fig. 14. A positive correlation was observed between flow rate and voltage.

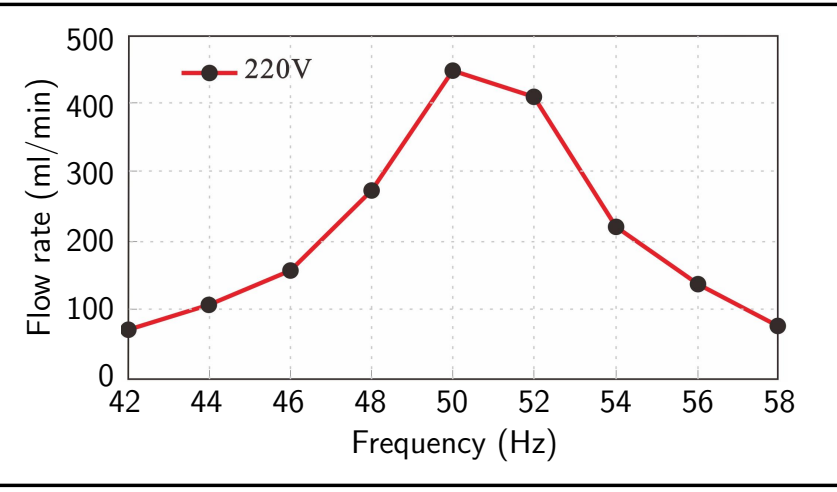

Figure 13. Frequency-dependent flow rate characteristics.

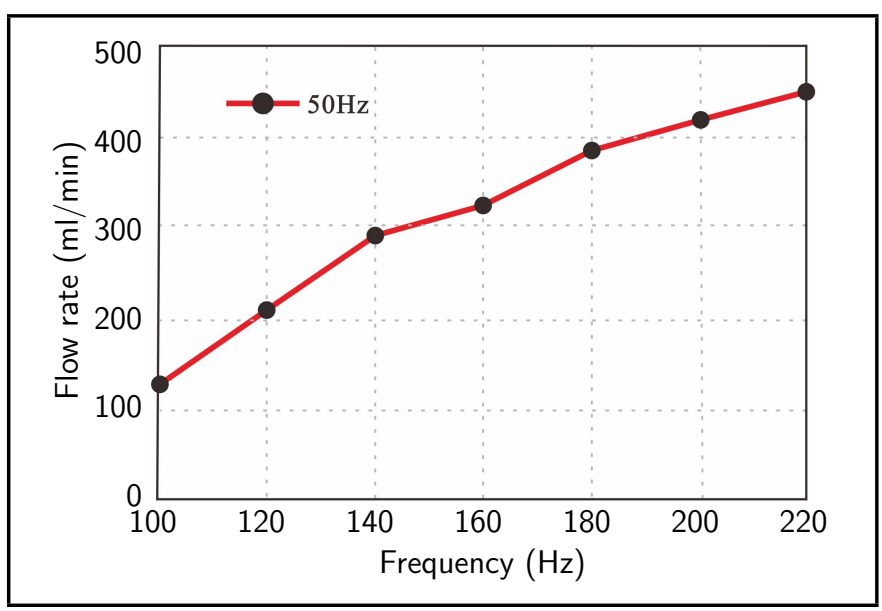

Figure 14. Voltage-dependent flow rate characteristics.

\section{CONCLUSION}

A resonant type piezoelectric pump working under power frequency excitation was developed in this study. The pump does not require a specialized power supply, which simplifies the drive power supply and reduces the cost. When driven by a common power frequency power supply, the pump can directly work under a resonant state and produce high flow rate. The experimental results show that the thickness of the TFV exerts the most significant effect on flow rate, followed by the material of the diaphragm and the quantity of inlet valve. On the basis of the optimized results, a prototype piezoelectric pump with a TFV thickness of $1.0 \mathrm{~mm}$, a pump chamber diaphragm of PDMS and one inlet valve was built. Driven by a power frequency power supply, the flow rate of the prototype can reach $441.77 \mathrm{ml} / \mathrm{min}$.

Although the new type piezoelectric pump successfully simplifies the drive power supply, some shortcomings need to be improved. Frequency drift and unstable flow were observed in the experiments. Thus, the robustness of the piezoelectric pump system should be strengthened. The future studies will focus on the thickness of TFV that is greater than $1.0 \mathrm{~mm}$. Miniaturisation of the TFV will also be challenging. In addition, the improvement of matching effect of the TFV and pump chamber will be beneficial for obtaining the best working condition of the pump. 


\section{ACKNOWLEDGEMENT}

This work was supported in part by the Project of National Natural Science Fund of China under Grant 51705124, the Fundamental and Research Funds for the Central Universities under Grant JZ2018HGTB0259 and in part by the Project of National Natural Science Fund of China under Grant 52075518, 52075143, 51975178.

\section{REFERENCES}

${ }^{1}$ Iverson, B. D. and Garimella, S. V. Recent advances in microscale pumping technologies: a review and evaluation, Microfluid Nanofluid, 5 (2), 145-174, (2008). https://dx.doi.org/10.1007/s10404-008-0266-8

${ }^{2}$ Laser, D. J. and Santiago, J. G. A review of micropumps, Journal of Micromechanics and Microengineering, 14 (6), 35-64, (2004). https://dx.doi.org/10.1088/0960$1317 / 14 / 6 / \mathrm{r} 01$

3 Woias, P. J. Micropumps—past, progress and future prospects, Sensors and Actuators. B: Chemical, 105 (1), 28-38, (2005). https://dx.doi.org/10.1016/j.snb.2004.02.033

${ }^{4}$ Pan, Q. S., He, L. G., Huang, F. S., Wang, X. Y., and Feng, Z. H. Piezoelectric micropump using dual-frequency drive, Sensors and Actuators A: Physical, 229, 86-93, (2015). https://dx.doi.org/10.1016/j.sna.2015.03.029

${ }^{5}$ Cheng, C. H. and Tseng, Y. P. Characteristic studies of the piezoelectrically actuated micropump with check valve, Microsystem Technologies, 19 (11), 1707-1715, (2013). https://dx.doi.org/10.1007/s00542-013-1857-1

${ }^{6}$ Kang, J. and Auner, G. W. Simulation and verification of a piezoelectrically actuated diaphragm for check valve micropump design, Sensors and Actuators A: Physical, 167 (2), 512-516, (2011). https://dx.doi.org/10.1016/j.sna.2011.01.012

${ }^{7}$ Nguyen, N.T. and Truong, T.Q. A fully polymeric micropump with piezoelectric actuator, Sensors and Actuators B: Chemical, 97 (1), 137-143, (2004). https://dx.doi.org/10.1016/s0925-4005(03)00521-5

8 Wang, X. Y., Ma, Y. T., Yan, G. Y., Huang, D. and Feng, Z. H. High flow-rate piezoelectric micropump with two fixed ends polydimethylsiloxane valves and compressible spaces, Sensors and Actuators A: Physical, 218, 94-104, (2014). https://dx.doi.org/10.1016/j.sna.2014.07.026

${ }^{9}$ Chen, J., Huang, D. and Feng, Z. H. A U-shaped piezoelectric resonator for a compact and high-performance pump system, Smart Materials and Structures, 24 (10), (2015). https://dx.doi.org/10.1088/0964-1726/24/10/105009

${ }^{10}$ Den Hartog, J. P. Mechanical vibrations. Dover publications. Inc, New York, (1985).
${ }^{11}$ Han, S. M., Benaroya, H., and Wei, T. Dynamics of transversely vibrating beams using four engineering theories, Journal of Sound and Vibration, 225 (5), 935-988, (1999). https://dx.doi.org/10.1006/jsvi.1999.2257

12 Hida, H., Shikida, M., Fukuzawa, K., Murakami, S., Sato, K., Asaumi, K., Iriye, Y., and Sato, K. Development of Self-vibration/detection AFM Probe Using Quartz Tuning fork, Ieej Transactions on Electrical and Electronic Engineering, 4 (3), 378-385 (2009). https://dx.doi.org/10.1002/tee.20421

13 Castellanos-Gomez, A., Agraï, N. and Rubio-Bollinger, G. Dynamics of quartz tuning fork force sensors used in scanning probe microscopy, Nanotechnology, 20 (21), (2009). https://dx.doi.org/10.1088/0957-4484/20/21/215502

${ }^{14}$ He, L. G., Pan, C. L., Wang, H. B., and Feng, Z. H. Arbitrary periodical mechanical vibrations can be realized in the resonant state based on multiple tuning fork structure. Review of Scientific Instruments, 84 (9), (2013). https://dx.doi.org/10.1063/1.4820919

15 Giessibl, F. J. High-speed force sensor for force microscopy and profilometry utilizing a quartz tuning fork, Applied Physics Letters, 73 (26), 3956-3958, (1998). https://dx.doi.org/10.1063/1.122948

${ }^{16}$ Clubb, D. O., Buu, O. V. L., Bowley, R. M., and Nyman, R. Quartz Tuning Fork Viscometers for Helium Liquids, Journal of Low Temperature Physics, 136 (1-2), 1-13, (2004). https://dx.doi.org/10.1023/b:jolt.0000035368.63197.16

17 Pan, Q. S., Zhang, Q., Wang, H. B., Li, W. and Feng, Z. H. Piezoelectric linear motor using resonant-type clamping based on harmonic vibration synthesis, Mechatronics, 24 (8), 1112-1119, (2014). https://dx.doi.org/10.1016/j.mechatronics.2014.08.004

18 Otto, K. N. and Antonsson, E. K. Extensions to the Taguchi method of product design, Journal of Mechanical Design, 115 (1), (1993). https://dx.doi.org/10.1115/1.2919325

${ }^{19}$ Kackar, R. N. Off-line quality control, parameter design, and the Taguchi method, Journal of Quality Technology, 17 (4), 176-188, (1985). https://dx.doi.org/10.1007/978-14684-1472-14

${ }^{20}$ Yang, W. H. and Tarng, Y. S. Design optimization of cutting parameters for turning operations based on the Taguchi method, Journal of Materials Processing Technology, 84 (1-3), 122-129, (1998). https://dx.doi.org/10.1016/S09240136(98)00079-X

21 Taguchi, G. Y. W. Introduction to off-line quality control, Central Japan Quality Control Assoc., Tokyo, (1979). 